Published in final edited form as:

Br J Sports Med. 2018 August ; 52(15): 1011-1012. doi:10.1136/bjsports-2018-099148.

\title{
Lowest perceived exertion in the late morning due to effects of the endogenous circadian system
}

\author{
Saurabh S Thosar ${ }^{1}$, Maya Xolal Herzig ${ }^{1}$, Sally A Roberts ${ }^{1}$, Alec M Berman ${ }^{1}$, Noal A \\ Clemons $^{1}$, Andrew W McHill ${ }^{1}$, Nicole P Bowles ${ }^{1}$, Miki Morimoto ${ }^{1}$, Matthew P Butler ${ }^{1}$, \\ Jonathan S Emens ${ }^{1,2}$, Steven A Shea ${ }^{1}$ \\ ${ }^{1}$ Oregon Institute of Occupational Health Sciences, Oregon Health \& Science University, Portland, \\ Oregon, USA
}

${ }^{2}$ Portland VA Medical Center, Portland, Oregon, USA

\section{INTRODUCTION}

There are daily variations in the rate of perceived exertion (RPE) ${ }^{12}$ during exercise, with lower RPE in the beginning of the night compared with the early morning. We studied whether RPE is affected by the internal circadian system while controlling for any effects of behavioural patterns, including sleep, activity and meals.

\section{METHODS}

Ten healthy adults (six females, aged $52 \pm 2$ years $($ mean \pm SEM) $)$ participated in a forced desynchrony protocol in dim light where all behaviours were evenly spread across the circadian cycle (figure 1A). ${ }^{3}$ After a normal night of sleep and baseline testing, participants underwent 10 recurring 5-hours 20-min of 'behavioural cycles' of 2-hours 40-min of sleep opportunities and 2-hours 40-min of standardised waking episodes. ${ }^{3}$ Approximately 1 hour after each sleep episode, participants performed mild intensity cycle ergometer exercise for $15 \mathrm{~min}$ at $50 \%$ predicted maximal heart rate (Karvonen's formula ${ }^{4}$ ). The speed and resistance were identical across each cycling bout. Participants rated their exertion using the Borg RPE scale ${ }^{1}$ after 3, 8 and 13 minutes of exercise. Salivary melatonin was used as the circadian phase marker $\left(0^{\circ}=\right.$ the dim light melatonin onset $) .{ }^{5} \mathrm{RPE}$ data were normalised within each participant (Z-scored), sorted into $60^{\circ}(\sim 4$ hour) circadian phase bins and

\footnotetext{
Correspondence to Dr. Saurabh S Thosar, Oregon Institute of Occupational Health Sciences, Oregon Health \& Science University, Portland OR 97239, USA; thosar@ohsu.edu.

Contributors SST had full access to all the data in the study and takes responsibility for the integrity of the data and the accuracy of the data analysis. MXH, SAR, AMB, NAC, AWM, NPB, MM, MPB, JSE and SAS contributed substantially in the study design or data acquisition and analyses, drafting the manuscript for intellectual content and approving the final version. All authors take accountability for the integrity of this work.

Publisher's Disclaimer: Disclaimer The findings and conclusions of this study are those of the authors and do not necessarily reflect the views of Oregon Health \& Science University or the National Institutes of Health.

Competing interests None declared.

Ethics approval Oregon Health \& Science University IRB for human subjects research.

Provenance and peer review Not commissioned; internally peer reviewed.
} 
compared across phases using repeated measures analysis of variance (online supplementary information).

\section{RESULTS}

The endogenous circadian system significantly affected RPE for the same workload with lowest RPE in the late morning (circadian phase $210^{\circ}, \approx 10: 45 \mathrm{AM}$ ) and highest RPE during the biological night $\left(90^{\circ}, \approx 3: 45 \mathrm{AM}\right)$. The magnitude of the circadian variation was $\sim 1 \mathrm{SD}$ (1 Z-score unit; figure 1B).

\section{DISCUSSION}

We have uncovered an endogenous circadian effect on RPE by strictly controlling and evenly distributing all behavioural patterns across the entire circadian cycle. Our discovery of lowest perceived exertion in the late morning suggests that exercise training may be easier at this time. We studied healthy middle-aged adults during mild exercise. If similar results prevail in athletes performing high-intensity exercise, this would lead to the intriguing possibility of optimising circadian phase of athletes (eg, with bright light) to the expected time of performance. ${ }^{6}$

\section{Supplementary Material}

Refer to Web version on PubMed Central for supplementary material.

\section{Funding}

Financial support: R01-HL125893 (SAS), F32-HL131308, National Space Biomedical Research Institute through NCC 9-58 and Medical Research Foundation of Oregon (SST), F32DK107146 (AWM), American Sleep Medicine Foundation (MPB), Ford Foundation (NPB) and Oregon Institute of Occupational Health Sciences and CTSA grant (UL1TR000128).

\section{REFERENCES}

1. Borg GA. Psychophysical bases of perceived exertion. Med Sci Sports Exerc1982;14:377-81. [PubMed: 7154893]

2. Faria IE, Drummond BJ. Circadian changes in resting heart rate and body temperature, maximal oxygen consumption and perceived exertion. Ergonomics1982;25:381-6. [PubMed: 7128568]

3. Butler MP, Smales C, Wu H, et al.The Circadian System Contributes to Apnea Lengthening across the Night in Obstructive Sleep Apnea. Sleep2015;38:1793-801. [PubMed: 26039970]

4. Karvonen MJ, Kentala E, Mustala O. The effects of training on heart rate; a longitudinal study. Ann Med Exp Biol Fenn1957;35:307-15. [PubMed: 13470504]

5. Benloucif S, Burgess HJ, Klerman EB, et al.Measuring melatonin in humans. J Clin Sleep Med2008;4:66. [PubMed: 18350967]

6. Song A, Severini T, Allada R. How jet lag impairs Major League Baseball performance. Proc Natl Acad Sci U S A2017;114:1407-12. [PubMed: 28115724] 

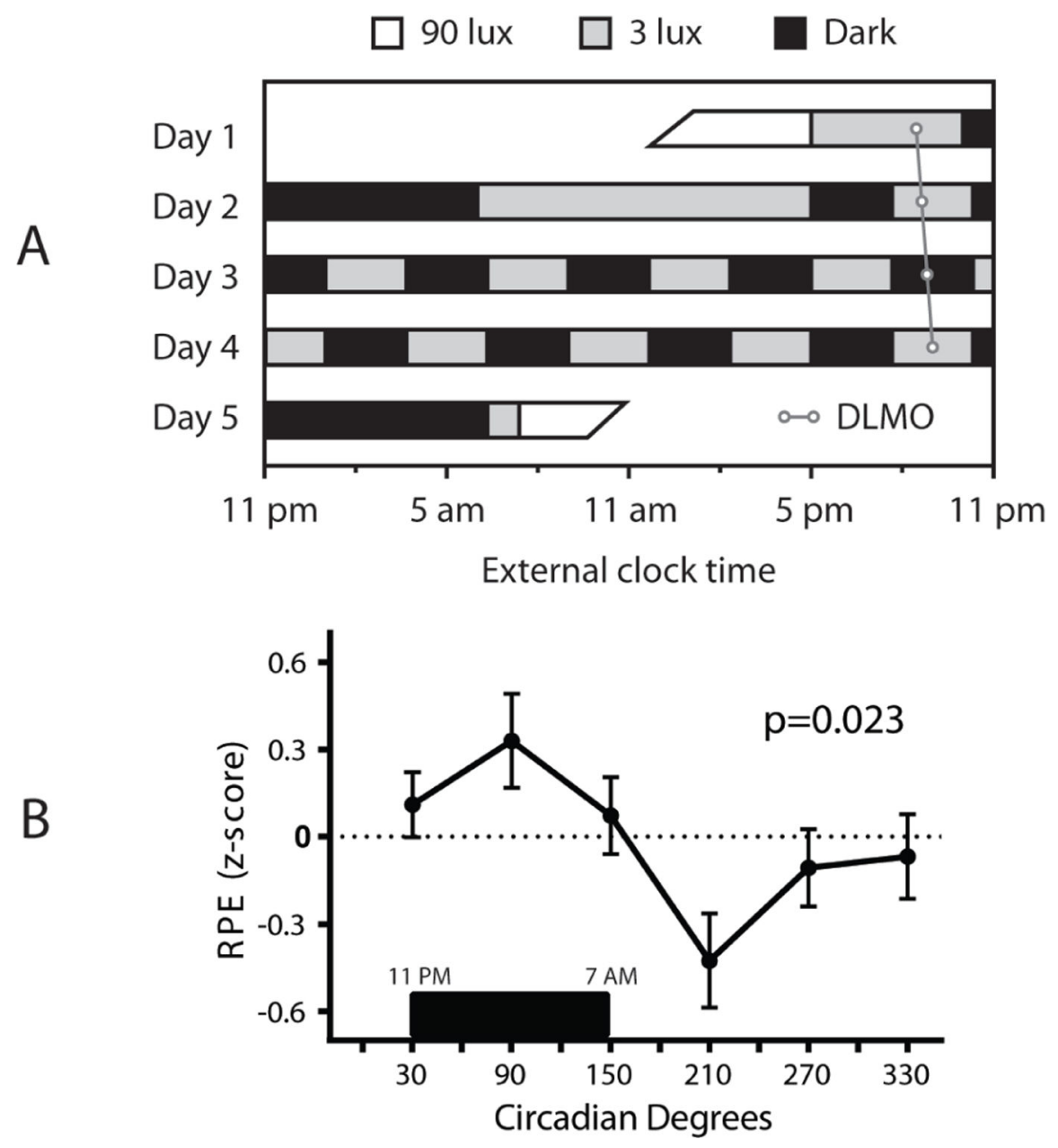

Figure 1.

(A) Schematic of five days 5 hours 20 min of forced desynchrony protocol. Grey bars represent wakefulness in dim lighting (<3 lux), while black bars represent sleep opportunities ( $<0.1 \mathrm{lux}$ ). Rate of perceived exertion (RPE) was assessed during the same levels of mild intensity exercise performed during each standardised wake period. Circadian phase was assigned based on time of the dim light melatonin onset (DLMO) from salivary samples, indicated by open circles. The diagonal line through the DLMOs represents the free running rhythm of the central circadian clock in dim light. (B) A significant circadian rhythm of RPE, during identical exercise bouts, with greatest exertion during the biological night and least exertion in the late morning. Black bar represents the average times when sleep normally occurs in these participants (11 PM - 7 AM). 